These discussions relate to theMarch 1990 JAOA quiz.

1 (b) False. Although immunofluorescence procedures offer a way to rapidly diagnose a change in cytoskeletal architecture, they are not currently being used to diagnose cases of Huntington's chorea. For differences to be detected to make early diagnosis possible requires implementation of a more defined approach and careful handling of the cells.

2. (c) Although many theories have been put forth for the etiology of endometriosis, ovarian follicle metaplasia is not a popular one. The four most popular are extravasation of endometrial cells through the fallopian tubes, hematogenous spread of en- dometrial cells, lymphatic spread of endometrial cells, and coelomic metaplasia of peritoneal mesothelium.

3. (d) Endometriosis is not common in postmenopausal women, but rather it is common in women of reproductive age and associated with an increased incidence of infertility and spontaneous abortion.

4. (c) Congenital dacryocystocele results from obstruction of the lacrimal apparatus. It usually resolves spontaneously $(80 \%)$ but may rarely require surgical intervention. They may also become infected. Both computed tomography and magnetic resonance imaging delineate the distended lacrimal sac and any herniation (if present) of the sac into the nasal cavity.

5. (a) Greater than $50 \%$ of cases of actinomycosis are located in the cervicofacial region, hence the name "lumpy jaw." One fourth of the infections occur in the abdominal region. Thoracic involvement occurs $15 \%$ of the time. Other regions are involved only sporadically.

6. (a) Although other antibiotics can be useful for the treatment of actinomycosis, the drug of choice is penicillin, which must be given for several months to ensure total eradication of the slow-growing Actinomyces organism.

\title{
Official call
}

\section{To the officers and members of the American Osteopathic Association:}

You are hereby notified that the annual meeting of the American Osteopathic Association will be held in Chicago, Illinois, July 11-15 at the Chicago Marriott Hotel.

The opening session of the annual meeting of the Board of Trustees will be held at 9 AM on Wednesday, July 11.

The House of Delegates will convene for the annual business session of the association at 2 PM on Friday, July 13. All meetings of the House of Delegates will be held at the Chicago Marriott Hotel. The Committee on Credentials will register delegates and alternate delegates beginning at 12:30 PM on Friday, July 13 . The House will conclude its session on Sunday, July 15.

At least 30 days prior to the first day of the annual meeting, the secretary of each divisional society must certify to the Executive Director of the American Osteopathic Association a list of the names and addresses of delegates and alternate delegates.

William H. Voss, DO, President

T. Eugene Zachary, DO, Speaker of the House 


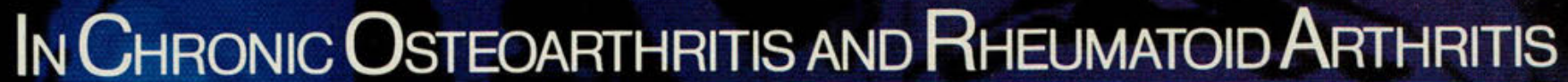

\section{NAPROSYN(NAPROXEN) CONCENIRATES HERE.}

decompression. Supplied by David W. Stoller, MD, Philipp Lang and Dimensional Medicine, Inc.

\section{Your experience and hundreds of clinical studies confirm:}

- Proven G.I. tolerability ${ }^{12^{*}}$

- An excellent renal and hepatic profile ${ }^{3, A^{* *}}$

\#1 NSAID brand prescribed for elderly patients ${ }^{\dagger+t}$

CONCENTRATES IN THE JOINT-LOW RISK TO KEY ORGANS*
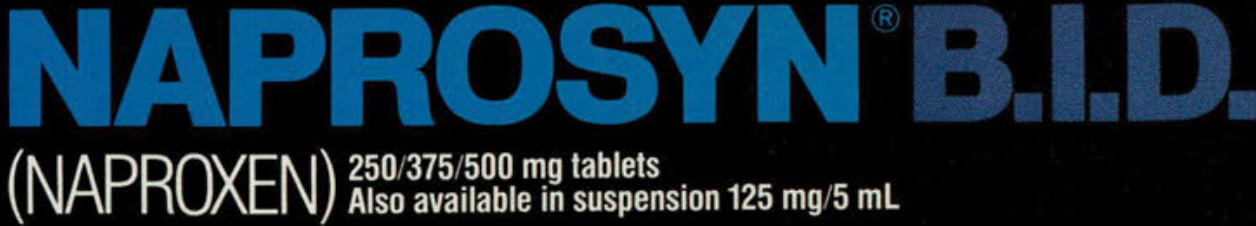

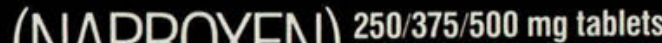

Also available in suspension $125 \mathrm{mg} / 5 \mathrm{~mL}$

"The most frequent complaints are gastrointestinal. See "Warnings" and "Precautions" sections of prescribing information *As with other NSAIDs, rare hepatic and renal reactions have been reported. Please see "Precautions section of prescribing information. $t$ it is prudent to use the lowest effective dose in the elderly 

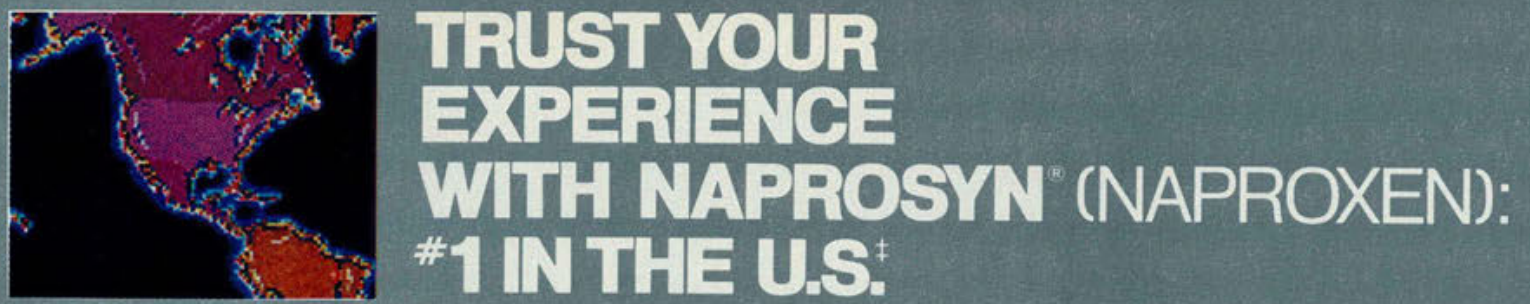

13 years of U.S. clinical experience

\#1 choice of rheumatologists and orthopaedic surgeons $\$$

- Over 7.5 billion patient therapy days worldwide

Wide range of strengths and forms:

\begin{tabular}{|c|c|c|c|c|c|c|c|}
\hline 0 & $\begin{array}{l}250 \mathrm{mg} \\
\text { tablets }\end{array}$ & somes & $\begin{array}{l}375 \mathrm{mg} \\
\text { tablets }\end{array}$ & eneses & $\begin{array}{l}500 \mathrm{mg} \\
\text { tablets }\end{array}$ & $=$ & $\begin{array}{l}\text { Suspension } \\
125 \mathrm{mg} / 5 \mathrm{~mL} \\
\text { (supplied in } 16 \mathrm{oz} \\
\text { unit-of-use bottles) }\end{array}$ \\
\hline
\end{tabular}

\section{CONCENTRATES IN THE JOINT-LOW RISK TO KEY ORGANS N/P $A P$
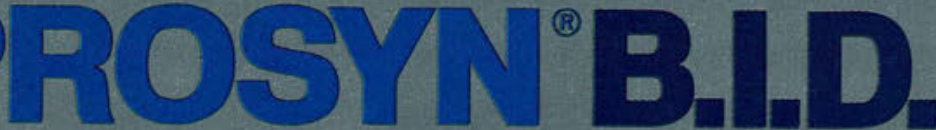 \\ (NAPROXEN) $)_{\text {Also available in suspension } 125 \mathrm{mg} / 5 \mathrm{~mL}}^{25037 / 500 ~}$}

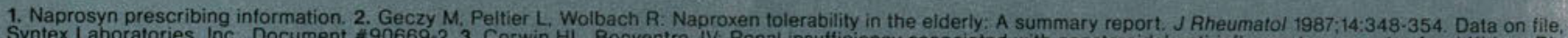

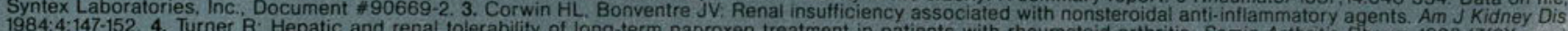

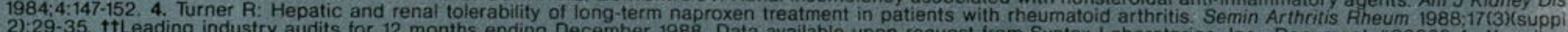

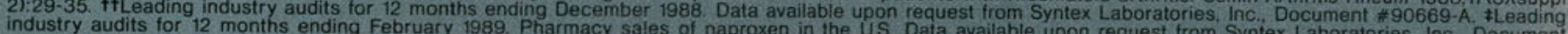

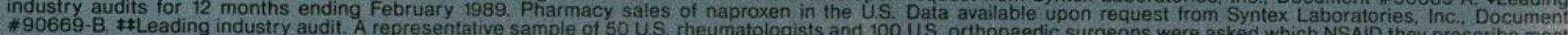

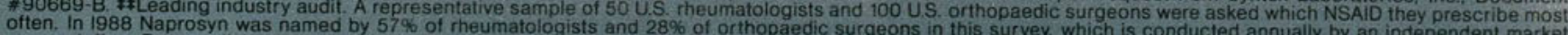
research firm. Data available upon request from Syntex Laboratories, Inc., Document \#90669-C
resten

Briel Summary:

Contraindications: Patients who have had allergic reactions to NAPROSYN. ANAPROX or ANAPROX DS or in whom aspirin or other NSAIDs induce the syndrome of asthma, rhinitis, and nasal polyps. Because anaphiylactic reactions usuany occur in patients with a history of such reactions, question patients for symptoms occur 0 s, uricarla, and hypotension associaied whth Nsali s betore starting therapy II such pertoration can occur at any the drug Warnings. Strious gi foxichly such as bieeding ulceration, and with NSAIDs. Remain alert for ulceration and bleed warning symptoms, in patients treated chronically. Gil tract symptoms in clinical trials symntomatic apoer Gi weers, grose bieding or pertoration unous to occur in aporoximately $1 \%$ of patients treated for 3.6 months, and in about $2-4 \%$ of natiants treated for one year. Inform patients about the sinns and/or symntoms of serinus fir toxicity and what stips to take if they occur. Studies have not identified any subset of patients not at risk of cheveloping peptic ulceration and bleeding. Except for a prior history of serious Gl events and other risk factors knowri to be associated with peptic vicer disease, such as aicoholism. smoking etc no risk factors (e.q. age sex) have been associated with increased risk. Elderly or debilitated patients seem to tolerate uceration or bleeding less well than others and most spontaneous reports of fatal Gt events are in this population. in considering the use of relatively large doses (within the recommended dosage range) sutficient benefit Should be anticipated to oftsef the potential increased risk of Gl toxicity. Precautions: DO NOT GivE
MAPROSYN- (NAPROXEN) CONCOMITANTLY WITH ANAPROX - (NAPROXEN SODIUM) OR ANAPROX*DS (NAPROXEN SODIUM) SINCE THEY CIRCULATE IN PLASMA AS THE NAPROXEN ANION. ACUT interstitial nephritis with hematuria, proteinuria, and nephrotic syndrome has been reported. Patients with impaired renal function, heart failure, liver dysfunction. patients taking diuretics, and the elderly are at greater risk of overt renal decompensation. If this occurs, discontinue the drug. Use with caution and monitor serum creatinine and/or creatinine clearance in patients with significantly impaired renal function. Use caution in patients with baseline creatinine clearance less than $20 \mathrm{~mL}$ minute. Use the lowest effective dose in the elderly or in patients with chronic alcoholic liver disease or cirrhosis. With NSAIDs, borderline elevations of liver tests may occur in up to $15 \%$ of patients. They may progress. remain unchanged, or be transient with continued therapy. Elevations of SGPT or SGOF occurred in controlled clinical trials in less than $1 \%$ of patients. Severe hepatic reactions, including jaundice and fatal hepatitis, have been reported rarely. If liver disease develops or if systemic manitestations occur le.9. eosinophita or rashi, drcontinue therapy. it steroid dosage is reduced or eiminated during therapy, do so slowiy and ouserve patients closely for adverse effects, including adrenal insufficiency and exacerbagrams or less who receive long-term therapy Peripheral edema caution in patients with fluid retention hypertension or heart talure The drug's antiprefore, use with

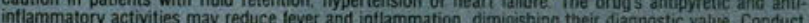
ophthalmic studies if any change or disturbance in vision occurs for patients with restricted conduct intake, note that the suspension contains $8 \mathrm{mg} / \mathrm{mL}$ of sodium. Information for Patients: S. NSAiUs can cause drcomiort and rareiy there are more cerious side eftecte such as GI bleedinn which may result in hospitalization and even fatal outcomes. Physicians may wish to discuss with patients the potential risks and likely benefits of NSAID treatment. particularly when they are used lor less serious conditions where treatment without NSAIDs may be an acceptable alternative. Patients should use caution for activities requiring alertness if they experience drowsiness, dizziness, vertigo or depression during therapy Laboratory Tests: Because serious gi tract ulceration and blecoing can occur without warning symptoms, follow chronically freated patients for signs and symptoms of these and inform them coumarin-type anticoagulants; a hydantoin. sulfonamide of caution when giving concomitantly with blockers; probenecid; or methotrexate. Drug/Laboratory Test Interactions: The drug may decrease platelet aggregation and prolong bleeding time or increase urinary values for 17-ketogenic steroids Jemporarily stop therapy for 72 hours before doing adrenal function tests. The drug may interfere with
urinary assays of 5 HIAM. Carcinogenesis: A 2-year rat study showed no evidence of carcinogenicity urinary assays of 5HIAA. Carcinogenesis: A 2-year rat study showed no evidence of carcinogenicity.
Pregnancy: Category B Do not use during pregnancy unless clearly needed. Avoid use during late Pregnancy: Category B Do not use during pregnancy unless clearly needed. Avoid use during late
pregnancy Nursing Mothers: Avoid use in nursing mothers. Pediatric Use: Single doses of $2.5-5 \mathrm{mg} / \mathrm{kg}$ with total daily dose not exceeding $15 \mathrm{mg} / \mathrm{kg}$ iday, are safe in children over 2 years of age. Adversi $1.500 \mathrm{mo}$ day than in those on $750 \mathrm{mg}$ day. In studies in children with iuvenile arthritis rash and prolonged bleeding times were more treguent GI and CNS reactione ahout the same and other manch less frequent than in adults Incidence Greater Than 1\%: Probable Causal Relationship: Gl: The most Irequent compiaints related to the GI tract: constipation; heartburn; abdominal pain; nausea; dyspep sia, diarmea, stomatitis. CNS: headache; dizziness: drowsiness: light-headedness, vertigo Dermato logic: itching (pruritus): skin eruptions, ecchymoses; sweating, purpura. Special Senses: tinnitus. hearing disturbances, visual disturbances. Cardiovascular: edema; dyspnea; palpitations. General bis.

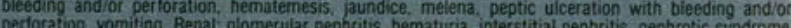

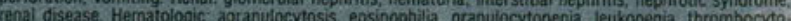
penia. CNS: depression, dream abnormalities, inability to concentrate insomnia malaise myalocia and muscle weakness. Dermatologic: alopecia, photosensitive dermatitis, skin rashes Speciat Senses- hearing imnairment. Cardiovascular: conoestive heart failure Respiratory ensinonhilic pneumont hearerat: anaphylactoid reactions. menstrual disorders, pyrexia (chills and fever). Causal Relationship Unknown Hematoiogic anlastic anemia nemoiytic anemia CNS connitive dystunction Dermionship epidermal necroiysis, erythema multiforme. Stevens-Johnson syndrome, urticaria. GI: non-peptic GI ulceration, uicerative stomatitis. Cardiovascular: vasculitis. General: angioneurotic edema, hyperglycemia, hypoglycemia. Overdosage: May have drowsiness, heartburn, indigestion, nausea, vomiting Empty stomach and use usual supportive measures. In animals $0.5 \mathrm{~g} / \mathrm{kg}$ of activated charcoal reduced plasma levels of naproxen. Caullon: Federal law prohibits dispensing without prescription. See package insert
for fuil Prescribing Information.

Rev 37 


\section{THE SHAPE OF THINGS TO COME FOR MILLIONS OF WOMEN}

\section{..IF OSTEOPOROSIS ISN'T TREATED}



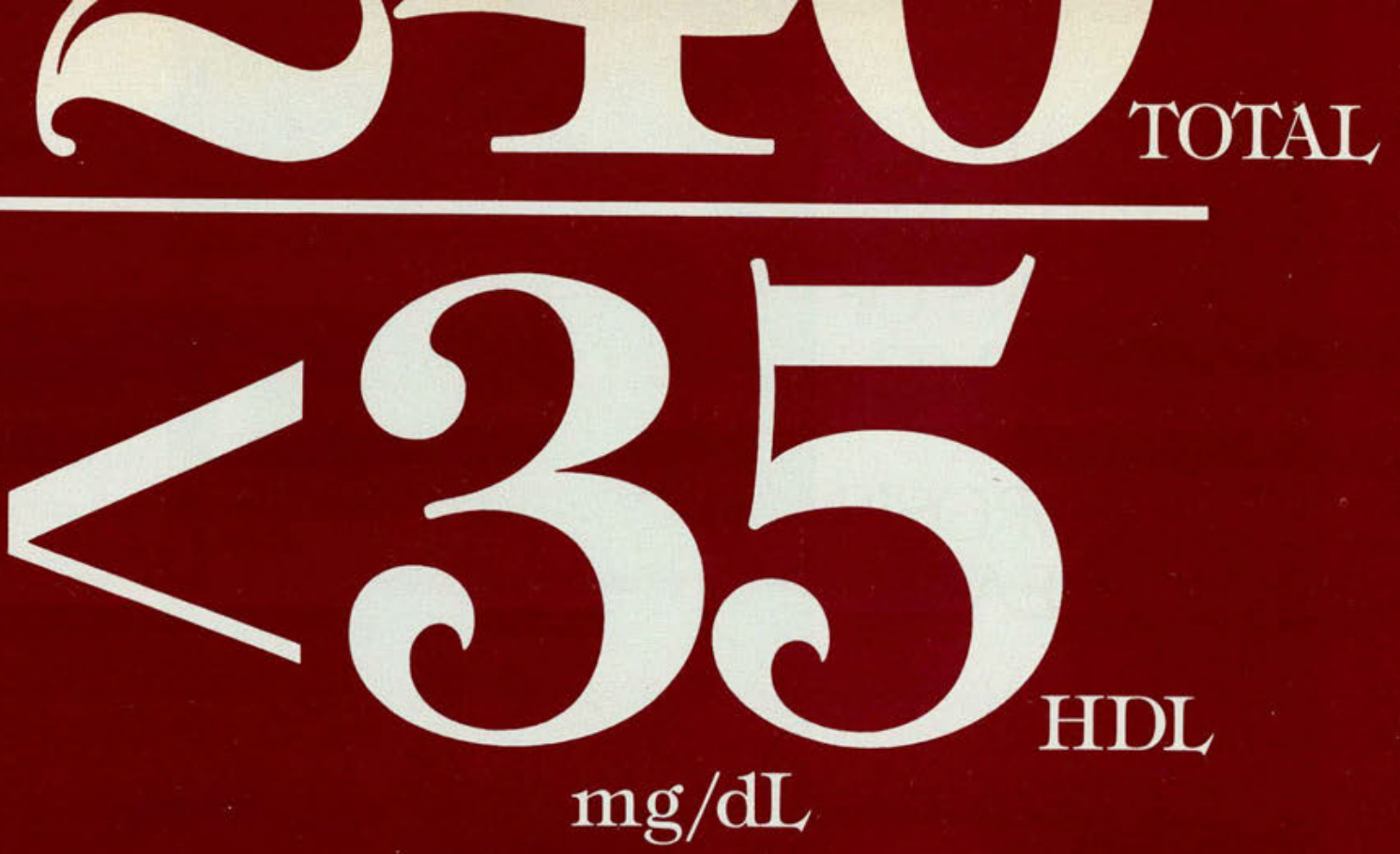

What's a common denominator of most heart attack victims?

Mixed hyperlipidemias-elevated cholesterol and triglycerides-are common among heart attack victims, ${ }^{1}$ and nearly two-thirds of people who developed myocardial infarction in the PROCAM Trial had a low $(<35 \mathrm{mg} / \mathrm{dL})$ baseline level of HDL cholesterol. ${ }^{2}$

HEART ATTACK PATIENTS (PROCAM TRIAL) ${ }^{2}$

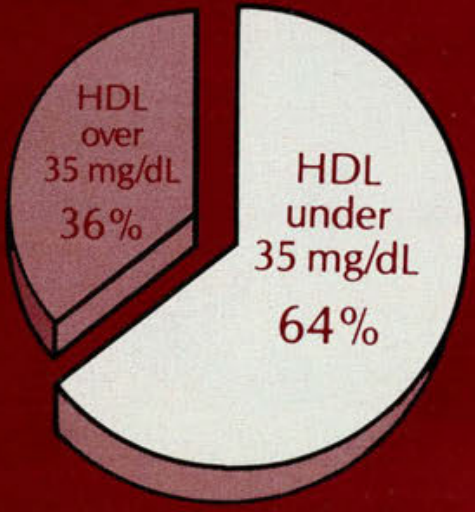




\section{A powerful case for}

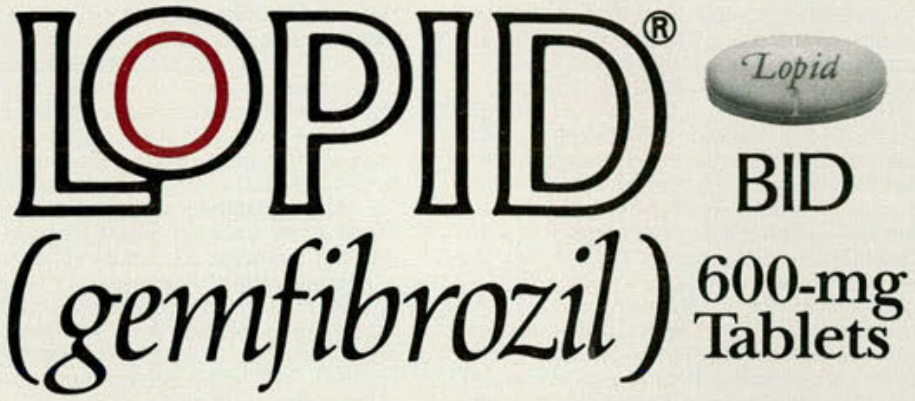

\section{Raised low HDL 25\%}

-in patients whose baseline HDL was below

$35 \mathrm{mg} / \mathrm{dL}$ in the landmark Helsinki Heart Study (HHS). ${ }^{3}$

\section{Reduced heart attack incidence* up to $62 \%$}

-in these HHS patients and $45 \%$ in HHS patients whose baseline HDL was below the median $(46.4 \mathrm{mg} / \mathrm{dL})$. Incidence of serious coronary events was similar for LOPID and placebo subgroups with baseline HDL above the median $(46.4 \mathrm{mg} / \mathrm{dL}){ }^{3}$

\section{Raised HDL levels $1 \frac{1}{2}$ to 3 times more effectively than lovastatin}

-in a 12-week, double-blind, randomized trial among patients with moderate to severe hyperlipidemia. Lovastatin achieved greater reductions in total serum cholesterol than gemfibrozil in this study population. ${ }^{4}$

\section{RAISES HDL DRAMATICALLY REDUCES HEART ATTACK}

LOPID is indicated for reducing the risk of coronary heart disease (CHD) in Type Ilb patients with low HDL, in addition to elevated LDL and triglycerides, and who have had an inadequate response to weight loss, diet, exercise, and other pharmacologic agents such as bile acid sequestrants and nicotinic acid.

*Defined as a combination of definite coronary death and/or definite myocardial infarction.

References: 1. Goldstein IL, Hazzard WR, Schrott HG, Bierman EL, Motulsky AG. Hyperlipidemia in coronary heart disease. I. Lipid levels in 500 survivors of myocardial infarction. I Clin Invest. 973;52:1533-153. 2. Assmann G, Schulte H. PROCAn 4. Tik Zunen MJ Helve E, Jäte treatment of primary hypercholesterolemia: the Finnish Multicenter Study. Am / Cardiol. 1988;62:35j-43j.

Please see last page of this advertisement for warnings,

contraindications, and brief summary of prescribing information. 
Lopid ${ }^{\circ}$ (Gemfibrozil Capsules and Tablets)

Before prescribing, please see full prescribing information.

A Brief Summary follows.

CONTRAINDICATIONS. 1. Hepatic or severe renal dysfunction, including primary biliary cirrhosis.

2. Preexisting gallbladder disease (See WARNINGS).

3. Hypersensitivity to gemfibrozil

WARINGS. 1. Because of chemical, pharmacological, and clinical similarities between gemfibrozil and clofibrate, the adverse findings with clofibrate in two large clinical studies may also apply to gemfibrozil. In the first of those studies, the Coronary Drug Project, 1000 subjects with previous myocardial infarction were treated for five years with clofibrate. There was no difference in mortality between the clofibrate-treated sub jects and 3000 placebo-treated subjects, but twice as many clofibrate-treated subjects developed cholelithiasis and cholecystitis requiring surgery. In the other study, conducted by the World Health Organization (WHO), 5000 subjects without known coronary heart disease were treated with clofibrate for five years and followed one year beyond. There was a statistically significant, $29 \%$, higher total mortality in the clofibrate treated than in a comparable placebo-treated control group. The excess mortality was due to a $33 \%$ increase in noncardiovascular causes, including malignancy, postcholecystectomy complications, and pancreatitis. The higher risk of clofibrate-treated subjects for gallbladder disease was confirmed.

During the Helsinki Heart Study and in the 11/2 year follow-up period since the tria was completed, mortality from any cause was $59(2.9 \%)$ in the Lopid group and 55 $(2.7 \%)$ in the placebo group. Mortality from any cause during the double-blind portion of the study was 44 deaths in the Lopid group and 43 in the placebo group. Because of the more limited size of the Helsinki Heart Study, this result is not statisticallysignificantly different from the $29 \%$ excess mortality seen in the clofibrate group in the separate WHO study. Noncoronary heart disease related mortality showed a $58 \%$ greater trend in the Lopid group ( 43 vs 27 patients in the placebo group, $p=0.056$ )

In the Helsinki Heart Study, the incidence of total malignancies discovered during the trial and in the $11 / 2$ years since the trial was completed was 39 in the Lopid group and 29 in the placebo group (difference not statistically significant). This includes 5 basal cell carcinomas in the Lopid group and none in the placebo group $(p=0.06$; historical data predicted an expected 4.7 cases in the placebo group). GI malignancies and deaths from malignancies were not statistically different between Lopid and placebo sub groups. Follow-up of the Helsinki Heart Study participants will provide further information on cause-specific mortality and cancer morbidity.

2. A gallstone prevalence substudy of 450 Helsinki Heart Study participants showed a trend toward a greater prevalence of gallstones during the study within the Lopid treatment group $(7.5 \%$ vs $4.9 \%$ for the place bo group, a $55 \%$ excess for the gemfibrozil group). A trend toward a greater incidence of gallbladder surgery was observed for the Lopid group ( 17 vs 11 subjects, a $54 \%$ excess). This result did not differ statistically from the increased incidence of cholecystectomy observed in the WHO study in the group treated with clofibrate. Both clofibrate and gemfibrozil may increase cholesterol excretion into the bile leading to cholelithiasis. If cholelithiasis is suspected, gallbladder studies are indicated. Lopid therapy should be discontinued if gallstones are found

3 . Since a reduction of mortality from coronary artery disease has not been

demonstrated and because liver and interstitial cell testicular tumors were increased in rats, Lopid should be administered only to those patients described in the INDICATIONS AND USAGE section. If a significant serum lipid response is not obtained, Lopid should be discontinued.

4. Concomitant Anticoagulants - Caution should be exercised when anticoagulants are given in conjunction with Lopid. The dosage of the anticoagulant should be reduced to maintain the prothrombin time at the desired level to prevent bleeding complications Frequent prothrombin determinations are advisable until it has been definitely determined that the prothrombin level has stabilized.

5. Concomitant therapy with Lopid and Mevacor (lovastatin) has been associated with rhabdomyolysis, markedly elevated creatine kinase (CK) levels and myoglobinuria, leading in a high proportion of cases to acute renal failure. In most subjects who have had an unsatisfactory lipid response to either drug alone, the possible benefit of combined therapy with lovastatin and gemfibrozil does not outweigh the risks of severe myopathy, rhabdomyolysis, and acute renal failure (See Drug Interactions). The use of fibrates alone, including Lopid, may occasionally be associated with myositis. Patients receiving Lopid and complaining of muscle pain, tenderness, or weakness should have prompt medical evaluation for myositis, including serum creatine kinase level determination. II myositis is suspected or diagnosed, Lopid therapy should be withdrawn

6. Cataracts - Subcapsular bilateral cataracts occurred in $10 \%$, and unilateral in $6.3 \%$ of male rats treated with gemfibrozil at 10 times the human dose

PRECAUTIONS. 1. Initial Therapy - Laboratory studies should be done to ascertain that the lipid levels are consistently abnormal. Before instituting Lopid therapy, every attempt should be made to control serum lipids with appropriate diet, exercise, weight los in obese patients, and control of any medical problems such as diabetes mellitus and

hypothyroidism that are contributing to the lipid abnormalities.

2. Continued Therapy-Periodic determination of serum lipids should be obtained and the drug withdrawn if lipid response is inadequate after 3 months of therapy.

3. Drug Interactions - (A) Lovastatin: Rhabdomyolysis has occurred with combined gemfibrozil and lovastatin therapy. It may be seen as early as 3 weeks after initiation of combined therapy or after several months. In most subjects who have had an unsatisfac tory lipid response to either drug alone, the possible benefit of combined therapy with lovastatin and gemfibrozil does not outweigh the risks of severe myopathy, rhabdomyolysis, and acute renal failure. There is no assurance that periodic monitoring of (B) Anticoagulants: CAUTION SHOULD BE EXERCISED WHEN ANTICOAGU. ANTS ARE GIVEN IN CONJUNCTION WITH LOPID. THE DOSAGE OF THE ANT COAGULANT SHOULD BE REDUCED TO MAINTAIN THE PROTHROMBIN TIME AT THE DESIRED LEVEL TO PREVENT BLEEDING COMPLICATIONS. FREQUENT PROTHROMBIN DETERMINATIONS ARE ADVISABLE UNTIL IT HAS BEEN DEFINITELY DETERMINED THAT THE PROTHROMBIN LEVEL HAS STABILIZED 4. Carcinogenesis, Mutagenesis, Impairment of Fertility-Long-term studie have been conducted in rats and mice at one and ten times the human dose. The incidence of benign liver nodules and liver carcinomas was significantly increased in high dose male rats. The incidence of liver carcinomas increased also in low dose males, but this increase was not statistically significant $(p=0.1)$. In high dose female rats, there was a significant increase in the combined incidence of benign, and malignant liver neoplasms. In male and female mice, there were no statistically significant differences
Lopid ${ }^{*}$ (Gemfibrozil Capsules and Tablets)

rom controls in the incidence of liver tumors, but the doses tested were lower than those mown to be carcinogenic with other fibrates

Male rats had a dose-related and statistically significant increase of benign Leydig cell tumors at 1 and 10 times the human dose

Electron microscopy studies have demonstrated a florid hepatic peroxisome proliferation following Lopid administration to the male rat. An adequate study to test for perox isome proliferation has not been done in humans but changes in peroxisome

morphology have been observed. Peroxisome proliferation has been shown to occur in humans with either of two other drugs of the fibrate class when liver biopsies were com. pared before and after treatment in the same individual.

Administration of approximately three or ten times the human dose to male rats for 10 weeks resulted in a dose-related decrease of fertility. Subsequent studies demonstrated that this effect was reversed after a drug-free period of about eight weeks, and it was not transmitted to the offspring

5. Pregnancy Category B-Reproduction studies have been performed in the rat at doses 3 and 9 times the human dose, and in the rabbit at 2 and 6.7 times the human dose. These studies have revealed no evidence of impaired fertility in females or harm to the fetus due to Lopid. Minor fetotoxicity was manifested by reduced birth rates observed at the high dose levels. No significant malformations were found among almost 400 offspring from 36 litters of rats and 100 fetuses from 22 litters of rabbits

There are no studies in pregnant women. In view of the fact that Lopid is tumorigenic in male and female rats, the use of Lopid in pregnancy should be reserved for those pafients where the benefit clearly outweighs the possible risk to the patient or fetus.

6. Nursing Mothers - Because of the potential for tumorigenicity shown for gemfibrozil in rats, a decision should be made whether to discontinue nursing or discontinue the drug, taking into account the importance of the drug to the mother

. Hematologic Changes - Mild hemoglobin, hematocrit and white blood cel decreases have been observed in occasional patients following initiation of Lopid therapy. However, these levels stabilize during long-term administration. Rarely, severe anemia, leukopenia, thrombocytopenia, and bone marrow hypoplasia have been reported. Therefore, periodic blood counts are recommended during the first 12 months op Lop administration.

8. Liver Function-Abnormal liver function tests have been observed occasionally during Lopid administration, including elevations of AST (SGOT), ALT (SGPT), LDH, bilirubin, and alkaline phosphatase. These are usually reversible when Lopid is discontinued. Therefore periodic liver function studies are recommended and Lopid therapy

terminated if abnormalities persist children have not been established. ADVERSE REACTIONS. In the double-blind controlled phase of the Helsinki Heart Study, 2046 patients received Lopid for up to 5 years. In that study, the following adverse reactions were statistically more frequent in subjects in the Lopid group (placebo incidence in parentheses): gastrointestinal reactions, $34.2 \%$ (23.8\%); dyspepsia, 19.6\% (11.9\%); abdominal pain, 9.8\% (5.6\%); acute appendicitis (histologically confirmed in most cases where data are available), $1.2 \%(0.6 \%)$; atrial fibrillation, $0.7 \%(0.1 \%)$

Adverse events reported by more than $1 \%$ of subjects, but without a significant difference between groups (placebo incidence in parentheses) were: diarrhea, $7.2 \%(6.5 \%)$ fatigue, $3.8 \%(3.5 \%)$; nausea/vomiting, $2.5 \%(2.1 \%)$; eczema, $1.9 \%(1.2 \%)$; rash, $1.7 \%$ $(1.3 \%)$; vertigo, $1.5 \%(1.3 \%)$; constipation, $1.4 \%(1.3 \%)$; headache, $1.2 \%(1.1 \%)$. Gallbladder surgery was performed in $0.9 \%$ of Lopid and $0.5 \%$ of placebo subjects, a $64 \%$ excess, which is not statistically different from the excess of gallbladder surgery observed in the clofibrate compared to the placebo group of the WHO study.

Nervous system and special senses adverse reactions were more common in the Lopid group. These included hypesthesia, paresthesias, and taste perversion. Other adverse reactions that were more common among Lopid treatment group subjects but where a causal relationship was not established include cataracts, peripheral vascular disease, and intracerebral hemorrhage

From other studies it seems probable that Lopid is causally related to the occurrence of musculoskeletal symptoms (See WARNINGS), and to abnormal liver function tests and hematologic changes (See PRECAUTIONS)

Reports of viral and bacterial infections (common cold, cough, urinary tract infections) were more common in gemfibrozil-treated patients in other controlled clinical trials of 805 patients. Additional adverse reactions that have been reported for gemfibrozil are listed below by system. These are categorized according to whether a causal relationship to treatment with Lopid is probable or not established:

CAUSAL RELATIONSHIP PROBABLE: Gastrointestinal: cholestatic jaundice; Centra/ Nervous System: dizziness, somnolence, paresthesia, peripheral neuritis, decreased libido, depression, headache; Eye: blurred vision; Genitourinary: impotence: Musculoskeletal: myopathy, myasthenia, myalgia, painful extremities, arthralgia synovitis, rhabdomyolysis (see WARNINGS and Drug Interactions under PRECAU TIONS); Clinical Laboratory: increased creatine phosphokinase, increased bilirubin, in creased liver transaminases (AST [SGOT], ALT [SGPT]), increased alkaline phosphatase; Hematopoietic: anemia, leukopenia, bone marrow hypoplasia, eosinophilia; Im . munologic: angioedema, laryngeal edema, urticaria; Integumentary: exfoliative dermatitis, rash, dermatitis, pruritus.

CAUSAL RELATIONSHIP NOT ESTABLISHED: General: weight loss; Cardiac: extrasys toles; Gastrointestinal: pancreatitis, hepatoma, colitis; Central Nervous System: confusion, convulsions, syncope; Eye: retinal edema; Genitourinary: decreased male fertility, Clinical Laboratory: positive antinuclear antibody; Hematopoietic: thrombocytopenia: mmunologic: anaphylaxis, Lupus-like syndrome, vasculitis; Integumentary: alopecia DOSAGE AND ADMINISTRATION. The recommended dose for adults is $1200 \mathrm{mg}$ administered in two divided doses 30 minutes before the morning and evening meal. MANAGEMENT OF OVERDOSE. While there has been no reported case of overdosage, symptomatic supportive measures should be taken should it occur.

References: 1. Frick MH, Elo O, Haapa K, et al: Helsinki Heart Study: Primary preven tion trial with gemfibrozil in middle-aged men with dyslipidemia. N Engl J Med 1987;317:1237-1245. 2. Manninen V, Elo O, Frick MH, et al: Lipid alterations and decline in the incidence of coronary heart disease in the Helsinki Heart Study. JAMA 1988; 260:641-651.3. Nikkila EA: Familial lipoprotein lipase deficiency and related disorders of chylomicron metabolism. In Stanbury J. B. et al. (eds): The Metabolic Basis of Inherited Disease, 5th ed., McGraw-Hill, 1983, Chap. 30, pp. 622-642.

PARKE-DAVIS

Morris Plains, NJ 07950 USA

$0737 \mathrm{G} 013$

PD-56-JA-5860-P-1A(10-89) 